\title{
Providing Services for Children With Developmental Difficulties, Delay or Disability - Early Diagnosis and Interventions at the Community Level
}

\author{
Roopa Srinivasan, Ashwini Marathe, Vibha Krishnamurthy \\ From Developmental Pediatrics and Early Childhood Development and Disability (ECDD) Services, Ummeed Child Development \\ Center, Mumbai, Mahar ashtra. \\ Correspondence to: Dr Ashwini Marathe, Ummeed Child Development Center, Ground Floor, Mantri Pride 1-B, 1/62, N.M. Joshi Marg, \\ Subhash Nagar,Lower Parel, Mumbai 400 011,Maharashtra.ummeed.ashwinimarathe@gmail.com
}

\begin{abstract}
This paper describes two programs using the recommended tiered approach - World Health Organization's (WHO) Nurturing Care Framework (NCF), viz., Early Childhood Champions (ECC) program and Child Development Aide (CDA) program delivered by Ummeed Child Development Center, a non-governmental organization in Mumbai. The ECC program builds capacity in community health workers (CHWs) employed by community based organizations in urban, semi-urban and rural areas to deliver the responsive caregiving and early learning components of WHO-NCF framework to all children (universal services) and those with or at risk for disabilities (targeted and indicated services). The CDA program trains $\mathrm{CHWs}$ to become disability workers in communities where rehabilitation services are scarce or nonexistent. ECC and CDA programs integrate ECD services into the existing work of established CHWs in the communities and have preliminary evidence of reach, effectiveness and acceptability. Till date, the $145 \mathrm{CHWs}$ trained by the ECC program have reached 7073 children, of whom $835(7.4 \%)$ have been identified with developmental delays. The ECC program meets the well-recognized need for training packages on responsive caregiving and early learning components of WHO NCF framework.
\end{abstract}

Keywords: Early childhood development, Non-governmental organization, Nurturing care framework, Child development center.

$\mathrm{T}$ he early years lay the foundation for health, wellbeing and learning throughout life. India has 164.5 million children under the age of six years [1], and many of them are at risk for suboptimal development due to factors such as poor nutrition, health and access to early learning environments. Recent literature has highlighted the high prevalence of disabilities in children under the age of five years in India [2]. The term developmental difficulties (DDs) is used in this article to denote developmental delays that are either acquired due to exposure to risk factors or that are present intrinsically from birth [3].

It is now well recognized that early childhood development (ECD) can be positively or negatively influenced by biological and psychosocial factors. Exposure to risk factors such as poor nutrition and low stimulation can negatively affect the development of typically developing children, and those born with disabilities. Long term outcomes can be positively influenced even for the most vulnerable children by intervening in the early years [4]. The World Health Organization's Nurturing Care Framework (WHO-NCF) provides a roadmap to help all children, including those with disabilities under three years of age, achieve their developmental potential. It includes components such as good health, adequate nutrition, responsive caregiving, security and safety and opportunities for early learning for all children, including those with DDs [3]. It recognizes that caregivers and children may have varied needs and recommends a tiered approach with varying intensity and range of services. This includes universal services for all children and targeted and indicated services for those with DDs.

India has a vast network of community health workers and one of the largest ECD programs in the world. The Integrated Child Development Scheme (ICDS) and the Rashtriya Bal Swasthya Karyakram (RBSK) are two of the government's flagship ECD programs [5]. The ICDS provides supplementary nutrition, growth monitoring, primary healthcare, referral to secondary healthcare and pre-school education to children under six years, while the RBSK serves children from birth to eighteen years of age, with a special focus on early intervention for children at risk because of the 4 Ds; defects at birth, deficiencies, diseases and developmental delays including disabilities $[5,6]$. These programs were set up to meet the needs of all children including those with DDs. Although the programs have achieved success addressing nutritional deficits, malnutrition and birth defects, challenges persist in addressing the developmental needs of children with DDs. Specifically, these are related to the non-availability of adequately trained personnel who can identify developmental difficulties at the community level, limited referral options on account of slow operationalization of District Early Intervention Centers (DEICs) and inadequate inter-program convergence and 
collaboration [7]. Many of these gaps can be addressed by offering training support to community programs run by the government and non-government organizations (NGOs).

This article provides a programmatic description of two community based ECD related programs implemented by Ummeed Child Development Center (https://ummeed.org/), an NGO with expertise in the area of child development and disability, the Early Childhood Champions (ECC) program and the Child Development Aide(CDA) Program.

\section{Early Childhood Champions (ECC) Program}

The ECC program builds capacity in community-based organizations $(\mathrm{CBO})$ in responsive caregiving and early learning components of the NCF. The objectives of the program are to evaluate needs of each community and tailor the ECC program to these needs, and to train community health workers to identify DDs and to offer individualized ECD support to all children (with and without DDs) from birth to six years of age in the community.

The ECC program trainers are psychologists, therapists and professionals trained in Early Childhood Education who in turn train community health workers (CHWs) within various CBOs. CBOs work with different communities to address issues that are important to people living in the community. Many of their staff members are from the community and have a firsthand experience of their felt needs [8].

The CBOs that were chosen for the delivery of ECC training had the following common criteria. They were serving low resource communities having a high prevalence of risk factors like malnutrition, anemia and alcoholism; the primary focus of their work was on maternal and child health or disability; and they had an existing cadre of CHWs willing to participate in the training (Web Table I). The geographical accessibility and funding status of the organization was also taken into consideration for long term engagement and sustainability of the program.

The ECC trainers meet with the senior management of the $\mathrm{CBO}$ to ascertain their commitment and interest in implementing ECD initiatives in the community. Further, a needs assessment through focus group discussions and in-depth interviews is conducted with various stakeholders (senior management of CBOs, local government officials, community leaders, community health workers, and families of young children). This helps the ECC program trainers to understand the needs of the community, scope and focus of existing programs and available resources. The ECC training has four modules of four days each, spread over one year, interspersed with time for implementation under mentorship support [9].

INDIAN PEDIATRICS
Module 1: CHWs are trained to know typical development of children under three years of age and to use the WHO Care for Child Development Intervention (CCDI) messages to promote ECD in all children [10].

Module 2: CHWs are trained to use International Guide for Monitoring Child Development (IGMCD) that has been previously standardized and validated for use in India [11]. It can be used by CHWs with low literacy levels or with limited formal training of child development, and in settings where direct access to health professionals may not be available. The tool is available in English, Hindi, Marathi and Gujarati.

By using the IGMCD, the CHW can understand how children between 0-42 months of age develop across the seven domains (expressive/receptive language, gross/fine motor, relating, play, and self-help) and identify protective and risk factors to development. Furthermore, the IGMCD has a built-in individualized ECD intervention component which helps $\mathrm{CHWs}$ provide nurturing care messages for each domain emphasizing the child's individual level of functioning, and identify next steps based on what the family is able to do. This approach is unique, because most other ECD interventions used in resource poor settings provide generic age-based messages, which may be less useful for children and families with unique needs [12]. The developmental progress of children is monitored through quarterly IGMCD administrations in the first year of life and then twice yearly. As such, the IGMCD permits longitudinal interventions with caregivers and children on different developmental trajectories and meets the need for individualized ECD intervention tools in low resource settings.

Module 3: It provides training in principles of responsive caregiving interventions outlined in Vroom [13]. Vroom is a program based on the principles of early brain development that helps parents boost their child's learning during the time they already spend together. It provides science-based tips and tools to inspire families to turn shared, everyday moments into 'brain building' moments. CHWs are trained to encourage play and communication activities of caregivers with the child along with opportunities for early learning, through the use of common household objects, homemade toys, reading and story-telling activities.

Module 4: The ECC trainers use the knowledge obtained from the preliminary needs assessment of the CBOs to understand the key resources and stakeholders in the community. This module equips the CHWs to identify common developmental disabilities and initiate simple interventions using their knowledge of locally available resources. 
In between modules, supervisors and CHWs engage with the families at a minimum of once a month, in the form of a home visit or group interactions. These visits help CHWs to reinforce the messages related to ECD, monitor the child's progress, teach the caregivers simple develop-mentally appropriate play based interventions and address caregiver concerns. The ECC trainers provide support and mentorship to the supervisors, who in turn support CHWs through regular visits to the field area, face-to-face meetings, and telephonic conversations.

Since its inception in 2015, 145 CHWs have been trained in the ECC program. The ECC trainers have collected quantitative data to track the reach (number of CHWs trained, number of children and families reached) and effectiveness of the program (no. of IGMCDs done and children with DDs identified) (Table I). Qualitative data from CHWs related to the acceptability of the training content is also collected by conducting focused group discussions (FGDs) with various stakeholders (senior management of CBOs, community leaders, community health workers, and families of young children).

\section{Child Development Aide (CDA) Program}

In many communities the presence of community workers who are now trained as Early Childhood Champions, makes them recognize the need for a trained disability resource person. The CDA program trains CHWs to become disability workers in communities where rehabili-tation services are scarce or nonexistent. The CDAs serve as a valuable community based resource, to provide interventions across developmental domains for children with DDs under the age of six years. The CDA program is an intensive one year training course for CHWs. It includes six months of didactic teaching with supervised practical experience of working directly with children and families at the center, followed by practical experience of working with children and families in the communities. The CHWs are mentored by the CDA trainers while they work in their own communities. This support is provided via phone/video calls and on-site visits as required. In the current SARS-CoV-2 pandemic, the CDA training has been modified to be implemented in a hybrid mode comprising offline, online and on-site training and mentorship.

Successful implementation of ECD programs in communities depends on two main factors. First, all stakeholders in the community must understand the factors that promote ECD for all children, including those with disabilities. Second, an evidence based, standardized ECD package that is responsive to the needs and resources available within each community must be used. In the absence of a standard package, WHO-NCF recommends delineating ECD programs into universal, targeted and indicated packages to respond to the specific needs of children at particular developmental risk or with developmental disabilities, with a focus on engaging parents in activities which promote development, rather than providing information on developmental milestones. It also recommends integrating ECD into existing services, as opposed to training a novel cadre of workers to deliver ECD. This strategy has been found to be cost effective, is recommended by NCF, and is also believed to promote intersectoral collaboration $[3,14]$.

The strength of the center's ECD program lies in incorporating many of these recommendations: It's universal, targeted and indicated packages respond to the specific needs of children at particular developmental risk, or with developmental disabilities as proposed by WHO-NCF. It supports the inclusion of children who are otherwise at the risk of not being reached by universal or conventional service models. It uses adult learning methods to deliver

Table I Reach and Effectiveness of Early Childhood Champions Program

\begin{tabular}{llllll}
\hline Organization & $\begin{array}{l}\text { CHWs trained } \\
n=145\end{array}$ & $\begin{array}{l}\text { Children reached } \\
n=1073\end{array}$ & $\begin{array}{l}\text { IGMCDs done } \\
n=11211\end{array}$ & $\begin{array}{l}\text { Delays } \\
\text { identified }^{a} \\
n=835\end{array}$ & $\begin{array}{c}\text { Home visits } \\
\text { received, } \\
n=39816\end{array}$ \\
\hline Sahayog trust & 25 & 1853 & 2662 & $166(12)$ & 13994 \\
Apanalaya & 24 & 922 & 974 & $116.2)$ & 3905 \\
Forbes Marshall -Pimpri & 9 & 439 & 1728 & $54(12.3)$ & 5636 \\
Forbes Marshall- Bopkhel & 9 & 332 & 528 & $35(5.5)$ & 1940 \\
Tara mobile créches & 20 & 980 & 711 & $410(23.9)$ & 7666 \\
SETCO foundation & 31 & 1715 & 3987 & $24(11.1)$ & $13(10.1)$ \\
Ambuja cement foundation & 14 & 293 & 492 & 129 & 7848 \\
Mumbai mobile créches & 13 & 539 & 1042 \\
\hline
\end{tabular}

Data presented as no. or ${ }^{a}$ no. (\%). CHWs-Community health workers; IGMCD-International guide for monitoring child development. 
standardized, evidence based universal, targeted and indicated packages to build caregiver skills for promotion of child development. The program offers strengths based mentoring to CHWs and their supervisors to maintain intervention quality and fidelity. The inclusion of a locally standardized and validated tool (IGMCD) for monitoring and supporting child development, adaptations of global curricula for parent training make it contextually appropriate. The modular nature of the center's ECC program lends it the flexibility to deliver content based on what the community is ready for, and its existing resources. Partnership with the CBOs to use their knowledge and expertise about the local community including key resources and stakeholders, has been a key feature of the program contributing to its acceptability. Qualitative data from parents of children in the community, and the CHWs and the ability of the CHWs to use the IGMCD to identify DDs indicates its acceptability and preliminary effectiveness respectively in rural and urban community settings.

The ability of the program to integrate ECD work into existing services offered by the CHWs is both a strength and a limitation. Without ongoing mentoring and support the CHWs can feel overburdened to take on ECD activities over and above their existing responsibilities. This is known to lead to attrition of CHWs or a diluted focus on ECD activities over time, thus negatively impacting sustainability [14]. Similarly, the built-in supervision and mentoring elements of the program help maintain fidelity but also make the programs more effort intensive and therefore less scalable.

\section{FUTURE DIRECTIONS}

The program developers recognize that working through the government system - whether through ICDS or the healthcare system can help ECD interventions to scale rapidly. The center's ECDD program is currently being piloted in one ICDS center in partnership with the local medical college. This presents an important opportunity for assessment of acceptability, feasibility and effectiveness of implementation in a government setting. This can potentially inform the need for adaptations to the program in the future to make it more sustainable and scalable.

Note: Supplementary material related to this manuscript is available at $w w w$. indianpediatrics.net

Contributors: RS,AM,VK: conception and design of the work, acquisition, interpretation of data; drafting the work and revising it critically for important intellectual content, final approval of the version to be published. All authors approved the final version of the manuscript, and are accountable for all aspects related to the study.

Funding: Trafigura Foundation; Porticus; H.T. Parekh Foundation and Schenker India Pvt. Ltd.

Competing interest: None stated.

\section{REFERENCES}

1. CensusInfo India 2011. Population size, 0-6 yrs (Number). Accessed April 20, 2021. Available from: https://censusindia. gov.in/2011census/censusinfodashboard/index.html

2. Olusanya BO, Davis AC, Wertlieb D, et al. Developmental disabilities among children younger than 5 years in 195 countries and territories, 1990-2016: A systematic analysis for the Global Burden of Disease Study 2016. Lancet Glob Heal. 2018;6:e110021.

3. World Health Organization, United Nations Children's Fund, World Bank Group. Nurturing care for early childhood development: a framework for helping children survive and thrive to transform health and human potential. Accessed April 20, 2021. Available from: https://apps.who.int/iris/bitstream/ handle/10665/272603/9789241514064-eng.pdf

4. Gertler P, Heckman J, Pinto R, et al. Labor market returns to an early childhood stimulation intervention in Jamaica. Science. 2014;344:998-1001.

5. Ministry of Woman and Child Development, Government of India. Integrated Child Development Services (ICDS) Scheme. Accessed April 20, 2021. Available from: https://icds-wcd. nic. in/icds.aspx

6. Ministry of Health \& Family Welfare, Government of India. Rashtriya Bal Swasthya Karyakram (RBSK). Accessed April 20, 2021. Available from: https://rbsk.gov.in/RBSKLive/

7. Deloitte India. National Communication Framework on RBSK. .Accessed April 20, 2021. Available from: https://nhm.gov.in/ images/pdf/programmes/RBSK/RBSK_IEC/RBSK_National Communication_Framework.pdf

8. IGNOU. Community Organisation: Concepts and Principles. Accessed June 07, 2021. Available from: http://ignou.ac.in/ upload/bswe-03-block1-unit-1-small-size.pdf

9. Wertlieb D, Krishnamurthy V. Special needs, special rights: addressing young children with disabilities via inclusive early childhood development. Early Childhood Matters. 2015;124: 59-64.

10. World Health Organization, UNICEF. Care for child development: improving the care for young children. Accessed April 20, 2021. Available from: http://www.who.int/maternal_ child_adolescent/documents/care_child_development/en/

11. Ertem IO, Krishnamurthy V, Mulaudzi MC, et al. Validation of the international guide for monitoring child development demonstrates good sensitivity and specificity in four diverse countries. Acta Paediatr. 2019;108:1074-86.

12. Ertem IO. The International guide for monitoring child development: Enabling individualised interventions. Early Childhood Matters. 2017;126:83-7.

13. Vroom. Hello, Brain Builder. Accessed April 20, 2021. Available from:https://www.vroom.org/

14. Kohli-Lynch M, Ponce HV, Bernal SR, et al. Human resources and curricula content for early child development implementation: Multicountry mixed methods evaluation. BMJ Open. 2020;10:e032134. 